J. Clin. Chem. Clin. Biochem.

Vol. 15, 1977, pp. 109-113

\title{
Erfahrungen mit einer neuen Glucosebestimmungsmethode auf dem Greiner Selective Analyzer II (GSA II)
}

\author{
Von E. Wasser \\ Aus der Medizinischen Klinik (Chefarzt: Prof. Dr. M. Schmid) des Stadtspiral Waid, Zürich
}

(Eingegangen am 25. Mai/15. November 1976)

Zusammenfassung: Die Hexokinasemethode und eine solche mit Glucosedehydrogenase wurden miteinander verglichen. Die Übereinstimmung war im allgemeinen sehr gut. Sodann wurde geprüft, ob Konservierungsmittel, Medikamente und Hämoglobin die Richtigkeit der Resultate von Patientenproben beeinflußten. Es wurde nichts nachteiliges festgestellt. Von den getesteten Kohlenhydraten reagierte 2-Desoxyglucose wie Glucose und kann zu falschen Werten führen. Abgesehen davon, ist diese eine verläßliche und kostengünstige Methode. Der Absorptions-Konzentrationsfaktor bleibt während mindestens 7 Tagen konstant bei einer Linearität zwischen 0,55 und 44,4 mmol/l Glucose.

\section{Investigation of a new method for the determination of glucose, using the Greiner Selective Analyzer II (GSA II)}

Summary: The hexokinase method and a method using glucose dehydrogenase are compared. In general, the two methods show good agreement. The samples were not deproteinized and were obtained from fasting patients of the medical, surgical, rheumatological and hemodialysis clinics. The possible influence of anticoagulants, preservatives and hemoglobin on the determination of glucose with glucose dehydrogenase was checked; there was no evidence of interference. Among the carbohydrates which were tested, only 2-deoxyglucose gave a positive reaction. In addition, this is a reliable and cheap method. The multiplication factor for the photometric analysis remains stable for seven days with a linearity between 0.55 and $44.4 \mathrm{mmol} / 1$ glucose.

\section{Einführung}

\section{Die Reaktion}

$$
D \text {-Glucose } \stackrel{\text { Glucosedehydrogenase } \left.{ }^{1}\right)}{\longrightarrow} \text { D-Gluconolacton }
$$

ist schon lange bekannt (1). Sie wurde nun auf dem GSA II ${ }^{2}$ ) als kinetische 2-Punkt-Methode eingesetzt $(2,3)$. Es galt zu untersuchen, ob die hier angewandte Reaktion ebenso spezifisch sei wie die Hexokinasemethode. Sodann wurde eine bessere Linearität bis in hohe Glucose-Konzentrationen angestrebt.

\section{Material und Methoden}

Die Hexokinasemethode und eine solche mit Glucosedehydrogenase wurden miteinander verglichen. Der Vergleich wurde an nicht enteiweißten Proben von Patienten aus der Medizinischen, Chirurgischen und Rheumaklinik, sowie aus der Hämodialysestation durchgeführt. Die Patienten befanden sich im nüchternen Zustand, und die Blutentnahme erfolgte durch Venenpunktion mit Kunststoffspritze.

\footnotetext{
1) Glucosedehydrogenase $=\beta$-D-Glucose: NAD-oxidoreductase EC 1.1.1.47.

2) Greiner Selective Analyzer II, Greiner Electronic SA, CH-4900 Langenthal.
}

\section{Glucosedehydrogenase-Methode}

Als Reagenzgrundlage dienten die Packungen Nr. 14051 (Phosphatpuffer) und 14053 (Enzymgemisch) der Firma Merck, Darmstadt. NAD (Nicotinamidadenindinucleotid) stammte von Boehringer, Mannheim (Produkt Nr. 15613). Pro 1 Liter Pufferlösung wurden 2 Flaschen Enzymgemisch und 1,6 g NAD gelöst. Es ergaben sich folgende Konzentrationen: Mutarotase ${ }^{3}$ ) $60 \mathrm{IU} / \mathrm{l}$ Glucosedehydrogenase $1733 \mathrm{IU} / \mathrm{l}$, NAD $2,4 \mathrm{mmol} / \mathrm{l}$, Phosphatpuffer $0,12 \mathrm{~mol} / \mathrm{l}, \mathrm{pH} 7,6$ bei $25^{\circ} \mathrm{C}$.

Die gebrauchsfertige Reagenzmischung ist während mindestens 7 Tagen stabil mit einem linearen Bereich $z$ wischen 0,55 bis $44,4 \mathrm{mmol} / 1$ Glucose.

\section{Berechnung}

[Glucose $]=\mathrm{dA} \times 555(1 \mathrm{Kommastelle})[\mathrm{mmol} / \mathrm{l}]$

\section{Hexokinase-Methode}

Die Hexokinasemethode wurde gemäß Angaben in der Methodensammlung des Geräteherstellers durchgeführt (4).

Sodann wurde geprüft, ob Antikoagulantien, Konservierungsmittel und Hämoglobin die Glucosedehydrogenasemethode beeinflussen.

3) Mutarotase $=$ Aldose-1 -epimerase EC 5.1.3.3. 


\section{Antikoagulantien}

Ethylendiaminte traessigsäure-di-Natriumsalz (Mcrck, Darmstadt), Ammoniumheparinat (Hoffmann-La Roche SA, Basel).

\section{Konservierungsmittel}

N-Ethylmaleinimid (Fluka AG, Buchs), Jodessigsäure-Natriumsalz (Merck, Darmstadt), Benzoesäure (Merck, Darmstadt), Natriumfluorid (Merck, Darmstadt).

\section{Stammlösungen}

EDTA $270 \mathrm{mmol} / \mathrm{l}$, Ammoniumheparinat 4500 NIH-Einheiten/ml, Natriumfluorid $120 \mathrm{mmol} / \mathrm{l}$, Jodessigsäure-Natriumsalz $25 \mathrm{mmol} / \mathrm{l}, D$-Xylose $555 \mathrm{mmol} / \mathrm{l}$, Fructose $11,1 \mathrm{mmol} / 1$, Galactose $11,1 \mathrm{mmol} / 1$, Lactose $5,8 \mathrm{mmol} / \mathrm{l}$, Mannose 11,1 $\mathrm{mmol} / 1,2$-Desoxyglucose 6,1 mmol/1, Cephalotin-Natrium $50 \mathrm{mmol} / \mathrm{l}$, Natriumascorbat (Redoxon) $568 \mathrm{mmol} / 1$, Vitamin $B_{1}$ (als Hydrochlorid) $60 \mathrm{mmol} / \mathrm{l}$.

\section{Glucose-Bestimmung}

\section{Glucosedehydrogenase-Methode}

Zum Einsatz gelangen $10 \mu$ l unverdünnte Probe, welche mit $100 \mu$ l destilliertem Wasscr in das Reaktionsgefäß gespült wird. Die Reaktionstemperatur beträgt $37^{\circ} \mathrm{C}$. Die Reaktionsgefäße I und II enthalten nun $110 \mu$ l verdünnte Probe. Bei Station 81 (Abb. 1, 2) wird das Gefäß II mit $1000 \mu$ l Reagenz beschickt. Bei Station 8 wird das Gefäß I ebenfalls mit $1000 \mu$ l Reagenz beschickt. Station 0 ist die Meßposition, wo die Absorptionsdifferenz von Reaktionsgefäß II (= Vollwert) und Reaktionsgefäß I (= Leerwert) bei einer Wellenlänge von $365 \mathrm{~nm}$ gemessen wird. Sodann wird das Resultat in mmol/l Glucose ausgedruckt.

\section{Methodenvergleich}

Beim Methodenvergleich Hexokinase-Glucosedehydrogenase waren beide Verfahren gleichzeitig programmiert, so daß aus einer Probe zum selben Zeitpunkt 2 verschiedene Methoden ausgeführt werden können.

\section{Henctoock Groiner Electronle Solectlve Andyzer GSA II}

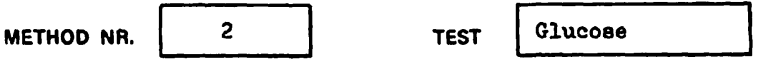

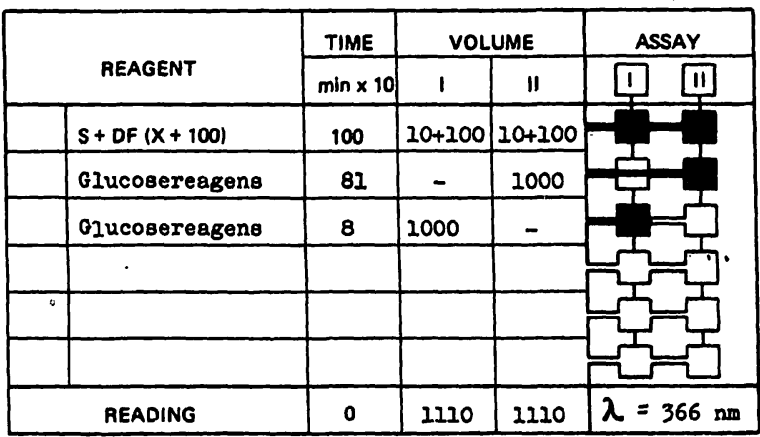

Abb. 1. Programmierschema des GSA II für die Volumina der Dosierer und deren Position.

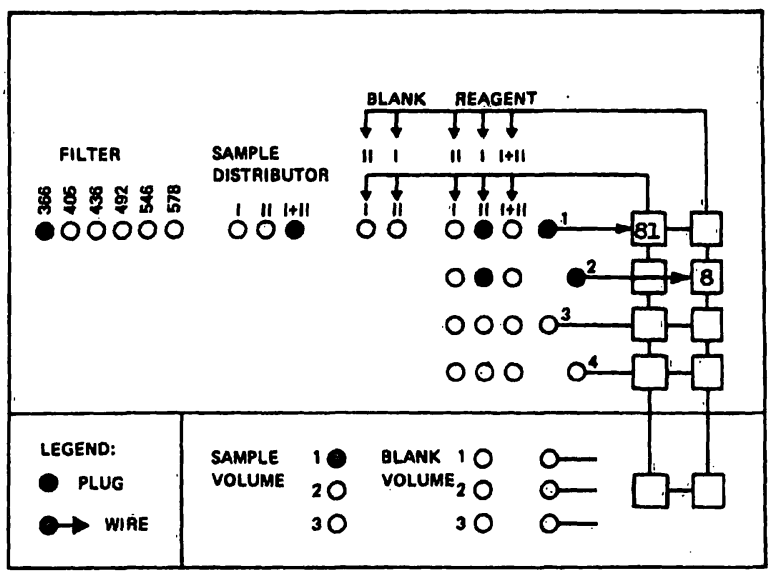

Abb. 2. Programmierschema des GSA Il für die Wahl des. Filters, des Leerwertes und der Dosierer.

\section{Ergebnisse}

\section{Line arität}

Die Linearität wurde mit wäßrigen Glucose-Lösungen geprüft. Wir verwendeten dazu wasserfreie Glucose ,Merck

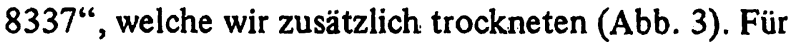
unseren Routinebetrieb setzen wir die obere Grenze der Linearität allerdings auf $44,4 \mathrm{mmol} / \mathrm{l}$, um etwaigem Aktivitätsverlust der Enzyme zu begegnen.

\section{Präzision}

Präzision von Tag zu Tag: $N=30 \quad \bar{x}=5,61 \quad s= \pm 0,193$

$$
\mathrm{VK}=3,45 \%
$$

Es gelangte ein Kontrollserum Ledernorm ${ }^{4}$ ) zur Anwendung. Dieses lyophilisierte Präparat wurde vorschriftsgemäß täglich rekonstituiert.

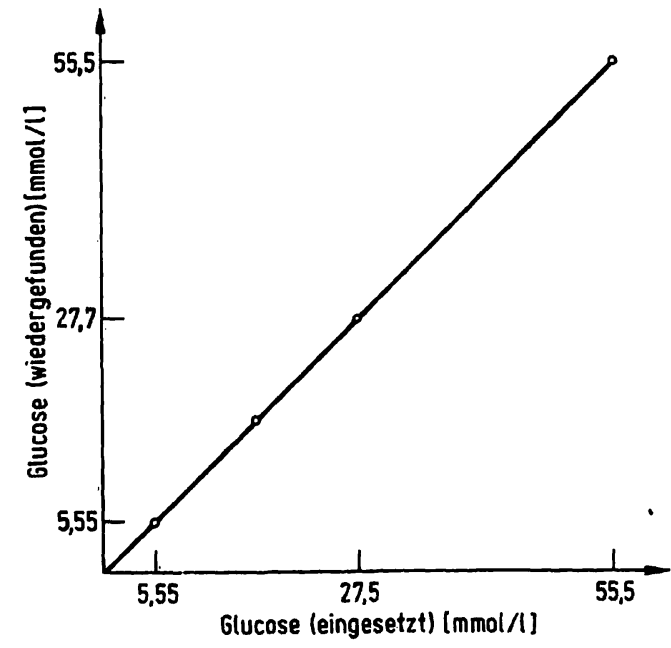

Abb. 3. Linearität der Glucosedehydrogenase-Methode auf dem GSA II. Die Standardlösungen wurden mit getrockneter . Glucose in gesättigter Benzoesäurelösung angesetzt.

4) Opopharma AG CH-8025 Zürich. 


\section{Kine tik der Reaktion}

Die beiden Meßreihen gegen dest. Wasser zeigen, daß während der Reaktionszeit das NAD nicht verändert wird, wenn keine Glucose vorhanden ist. Im Durchschnitt erreicht der Leerwert 16,8\% der Absorption des Vollwertes (Abb. 4).

Die Reaktion in Prozeßgefäß I verläuft also langsamer als in Prozeßgefäß II. Dies ist mit Sicherheit auf eine unterschiedliche Temperatur in den beiden Gefäßen zurückzuführen. Die Zeit von $48 \mathrm{~s}$ reicht nicht aus, um die Lösung im Leerwert auf $37^{\circ} \mathrm{C}$ zu bringen.

\section{Prüfung des Einflusses von Antikoagulantien (5) EDTA}

Die EDTA-Konzentration wurde absichtlich höher als üblich gewählt, weil die Blutentnahmegefäße eine gewisse Menge des Stoffes vorgelegt haben und die Röhrchen unter Umständen nur mit $1 \mathrm{ml}$ statt $5 \mathrm{ml}$ Blut gefüllt werden.

Bei allen diesen Zumischversuchen wurde der Verdünnungseffekt bei der Berechnung berücksichtigt.

$$
\begin{aligned}
& \mathrm{N}=32 \quad \mathrm{r}=0,998 \quad \mathrm{y}=-0,84+1,000 \mathrm{x} \\
& \bar{x}=6,61 \quad x \text { ohne EDTA } \\
& \bar{y}=6,54 \quad y \text { mit EDTA } \\
& s_{y x}= \pm 0,048
\end{aligned}
$$

\section{Ammoniumheparinat}

Dieses Antikóagulans ist in vielen kommerziell erhältlichen Blutentnahmegefäßen vorgelegt. Auch hier wurde die Konzentration etwa auf das fünffache der üblichen

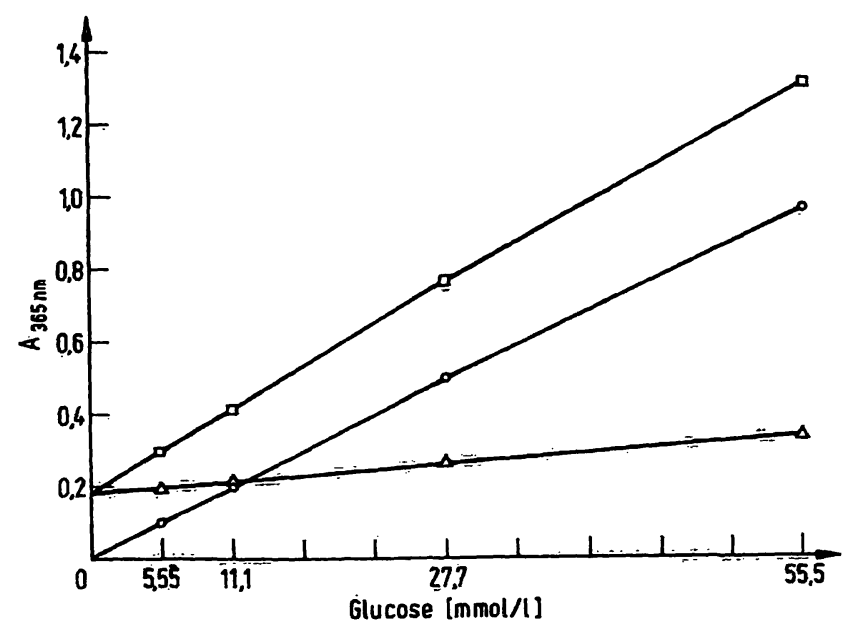

Abb. 4. Absorptionswerte im GSA II von Glucosestandardlösungen bestimmt mit der Glucosedehydrogenase= Methode.

$\Delta$ Inhalt von Prozeßgefäß I aus Dosierer 8 gegen dest. Wasser gemessen; Reaktionszeit $\mathbf{4 8} \mathrm{s}$. - Inhalt von Prozeßgefäß İ aus Dosierer 81 gegen dest. Wasser gemessen; Reaktionszeit $486 \mathrm{~s}$. o Absorptionsdifferenz der beiden Messungen. gesetzt, d. h. 225 NIH-Einheiten statt etwa 45 NIHEinheiten.

$$
\begin{aligned}
& N=32 \quad r=0,997 \quad y=4,07+0,964 x \\
& \bar{x}=6,43 \quad x \text { ohne Heparin } \\
& \bar{y}=6,41 \quad y \text { mit Heparin } \\
& s_{y x}= \pm 0,05
\end{aligned}
$$

\section{Konservierungsmittel}

\section{Natriumfluorid}

Natriumfluorid wird hauptsächlich dort verwendet, wo eine Vollblutprobe längere Zeit aufbewahrt werden muß, da es gleichzeitig die Glykolyse hemmt.

$$
\begin{aligned}
& N=20 \quad r=0,976 \quad y=0,101+0,956 x \\
& \bar{x}=6,29 \quad x \text { ohne } \mathrm{NaF} \\
& \bar{y}=6,11 \quad y \text { mit NaF } \\
& s_{y x}= \pm 0,094
\end{aligned}
$$

\begin{tabular}{|c|c|c|}
\hline $\begin{array}{l}\text { Jodacetat } \\
\text { [mmol/l] }\end{array}$ & $\begin{array}{l}\text { Glucose } \\
\text { [mmol/1] }\end{array}$ & $\begin{array}{l}\text { gefunden } \\
{[\%]}\end{array}$ \\
\hline $\begin{array}{l}0,0 \\
0,05 \\
0,1 \\
0,5\end{array}$ & $\begin{array}{l}27,5 \\
27,7 \\
27,4 \\
27,8\end{array}$ & $\begin{array}{c}100 \\
100,7 \\
99,1 \\
101\end{array}$ \\
\hline
\end{tabular}

\section{Jodessigsäure-Natriumsalz (6) (Tab. 1)}

Jodessigsäure kann NADH zerstören und die Wasserstoffübertragung verhindern. Die Konzentration im Reaktionsgemisch ist jedoch zu klein, um einen merklichen Einfluß auszuüben. Die Konzentration im Reaktionsgemisch beträgt in diesen Versuchen höchstens $4,5 \mu \mathrm{mol} / 1$.

Tab. 1. Einem Mischplasma wurden steigende Konzentrationen von Jodacetat zugefügt und dann der Glucosegehalt in Doppelbestimmungen bestimmt.

\section{N-Ethylmaleinimid (7) (Tab. 2)}

Dieser Glycolysehemmer gilt allgemein als am besten geeignet, weil er in keine Enzyme der Bestimmungssysteme eingreifen soll. Seine Wirksamkeit als Konservierungsmittel wurde schon von Kohler (7) 1963 uberpriift.

Tab. 2. Einem Mischplasma wurde N-Ethylmaleinimid in steigenden Konzentrationen zugefüt und dann die Glucose in Doppelbestimmungen bestimmt.

\begin{tabular}{lll}
\hline $\begin{array}{l}\text { N-Ethylmaleinimid } \\
{[\mathrm{mmol} / \mathrm{l}]}\end{array}$ & $\begin{array}{l}\text { Glucose } \\
{[\mathrm{mmol} / \mathrm{l}]}\end{array}$ & $\begin{array}{l}\text { gefunden } \\
{[\%]}\end{array}$ \\
\hline 0,0 & 16,33 & 100 \\
0,08 & 16,28 & 99,7 \\
0,8 & 16,44 & 100,7 \\
\hline
\end{tabular}


Beeinflussung der Meßergebnisse durch andere Kohlenhydrate

\section{$D$-Xylose (Tab. 3)}

Einerseits wurden wäßrige Lösungen von $D$-Xylose geprüft, ob sie eine der $D$-Glucose ähnliche Reaktion zeigen, andererseits wurden Zumischversuche durchgefuhrt mit einem Mischplasma. Auch bei einem Gehalt von 13,3 $\mathrm{mmol} / \mathrm{l}$ an $D$-Xylose wurde der Ausgangswert des Mischplasmas nicht verändert.

Nach unseren Modellversuchen im Spektralphotometer beträgt die Umsatzgeschwindigkeit von $D$-Xylose etwa $1 \%$ derjenigen von $D$-Glucose. Eine Störung der Glucosebestimmung durch Xylose während einer Xylose-Belastung ist somit kaum gegeben (8).

Tab. 3. Einem Mischplasma wurde Xylose in Konzentrationen, wie sie im „Xylose-Test“ vorkommen können, zugesetzt.

\begin{tabular}{lll}
\hline $\begin{array}{l}\text { Xylose } \\
{[\mathrm{mmol} / \mathrm{l}]}\end{array}$ & $\begin{array}{l}\text { Glucose } \\
{[\mathrm{mmol} / \mathrm{l}]}\end{array}$ & $\begin{array}{l}\text { gefunden } \\
{[\%]}\end{array}$ \\
\hline 0,0 & 6,67 & 100 \\
0,67 & 6,67 & 100 \\
3,3 & 6,67 & 100 \\
13,3 & 6,67 & 100 \\
\hline
\end{tabular}

\section{Andere untersuchte Zucker}

Bei den übrigen Zuckern, welche wir untersuchten, ist noch die Fructose zu erwähnen, welche auf dem GSA II keine Reaktion zeigte mit der GlucosedehydrogenaseMethode, mit der Hexokinase-Methode jedoch zu etwa 5\% umgesetzt wurde. Für dieses Phänomen ist der Isomerasegehalt der Hexokinase verantwortlich (s. Tab. 4).

Die 2-Desoxyglucose wird gelegentlich für den HollanderTest verwendet $(9,10)$. Wir fanden für die 2-Desoxyglucose mit Glucosedehydrogenase eine Umsatzrate von $107 \%$ verglichen mit Glucose. Steht ein Patient also unter 2-Desoxyglucose, so kann mit dieser Methode kein richtiger Glucosewert erhalten werden.

Tab. 4. Wäßrige Lösungen der Zucker wurden in Abwesenheit von Glucose geprüft, ob sie eine positive Reaktion ergeben.

\begin{tabular}{|c|c|c|}
\hline Zucker & "Glucose" & $\begin{array}{l}\text { gefunden } \\
\text { [mmol/1] }\end{array}$ \\
\hline $\begin{array}{l}\text { Fructose } \\
\text { Galactose } \\
\text { Lactose } \\
\text { Mannose } \\
D \text {-Xylose } \\
D \text {-Xylose } \\
\text { 2-Desoxyglucose }\end{array}$ & $\begin{array}{r}11,1 \\
11,1 \\
5,8 \\
11,1 \\
11,1 \\
555,5 \\
12,2\end{array}$ & $\begin{array}{l}0 \\
0 \\
0 \\
0,11 \\
0,11 \\
8,55 \\
13,0\end{array}$ \\
\hline
\end{tabular}

\section{Medikamente (11)}

Beim Testen von Medikamenten, ob sie chemische Reaktionen beeinflussen können, muß man sich bewußt sein, daß es sich um in vitro-Modellversuche handelt. Wir prüften 1 Antibiotikum und 2 Vitamine (Tab. 5, 6 und 7). Wenn auch mit diesem Verfahren keine Beeinflussungen festgestellt werden konnten, so wäre es möglich, daß Metabolite dieser Substanzen stören könnten.

Tab. 5. Einem Mischplasma wurden steigende Konzentrationen von Cephalotin-Natrium zugegeben und dann die Glucose bestimmt.

\begin{tabular}{lll}
\hline $\begin{array}{l}\text { Cephalotin-Natrium } \\
\text { [mmol/1] }\end{array}$ & $\begin{array}{l}\text { Glucose } \\
{[\mathrm{mmol} / \mathrm{l}]}\end{array}$ & $\begin{array}{l}\text { gefunden } \\
{[\%]}\end{array}$ \\
\hline 0 & 6,77 & 1.00 \\
0,12 & 6,77 & 100 \\
0,50 & 6,83 & 100,9 \\
2,5 & 6,77 & 100 \\
\hline
\end{tabular}

Tab..6. Einem Mischplasma wurden steigende Konżentrationen einer Natriumascorbatlösung zugegeben und dann der Glucosegehalt in Doppelbestimmungen bestimmt.

\begin{tabular}{lll}
\hline $\begin{array}{l}\text { Natriumascorbat } \\
{[\mathrm{mmol} / \mathrm{l}]}\end{array}$ & $\begin{array}{l}\text { Glucose } \\
{[\mathrm{mmol} / \mathrm{l}]}\end{array}$ & $\begin{array}{l}\text { gefunden } \\
{[\%]}\end{array}$ \\
\hline 0,0 & 7,1 & 100 \\
0,6 & 7,0 & 98,6 \\
1,2 & 7,2 & 101 \\
3,0 & 7,3 & 102,8 \\
\hline
\end{tabular}

Tab. 7. Einem Mischplasma wurden steigende Konzentrationen einer Vitamin- $B_{1}$-Lösung zugesetzt und danach der Glucosegehalt im Doppel bestimmt.

\begin{tabular}{lll}
\hline $\begin{array}{l}\text { Vitamin } B_{1} \\
{[\mathrm{mmol} / 1]}\end{array}$ & $\begin{array}{l}\text { Glucose } \\
{[\mathrm{mmol} / \mathrm{l}]}\end{array}$ & $\begin{array}{l}\text { gefunden } \\
{[\%]}\end{array}$ \\
\hline 0 & 7,1 & 100 \\
0,3 & 7,1 & 100 \\
0,6 & 7,0 & 98,6 \\
3,0 & 7,1 & 100 \\
\hline
\end{tabular}

\section{Hämoglobin}

Hämoglobin stört bis zu einer Konzentration von 0,6 mmol/1 Plasma die Bestimmungen nicht (Tab. 8, 9).

Tab. 8. Einem Mischplasma wurden steigende Mengen einer Hämoglobinlösung beigemischt und die Glucose in Doppelbestimmungen bestimmt.

\begin{tabular}{lll}
\hline $\begin{array}{l}\text { Hämoglobin } \\
\text { [mmol/1] }\end{array}$ & $\begin{array}{l}\text { Glucose } \\
{[\mathrm{mmol} / \mathrm{I}]}\end{array}$ & $\begin{array}{l}\text { gefunden } \\
{[\%]}\end{array}$ \\
\hline 0 & 11,8 & - \\
0,03 & 11,9 & 100,8 \\
0,06 & 11,8 & 100 \\
0,3 & 12,1 & 102,5 \\
0,6 & 12,1 & 102,5 \\
\hline
\end{tabular}


Tab. 9. Glucosebestimmung in wäßrigen Standardlösungen von Glucose, von denen jede einen Hämoglobingehalt von $0,07 \mathrm{mmol} / \mathrm{l}$ aufweist.

\begin{tabular}{lll}
\hline $\begin{array}{l}\text { Standardlösung } \\
\text { [mmol/1] }\end{array}$ & $\begin{array}{l}\text { Glucose } \\
{[\mathrm{mmol} / \mathrm{l}]}\end{array}$ & $\begin{array}{l}\text { gefunden } \\
{[\%]}\end{array}$ \\
\hline 0 & 0 & - \\
1,39 & 1,41 & 101,4 \\
2,78 & 2,70 & 97,1 \\
5,55 & 5,55 & 100 \\
11,1 & 12,2 & 109,9 \\
27,7 & 29,1 & 105,0 \\
55,5 & 54,4 & 98,0 \\
\hline
\end{tabular}

Vergleich der Glucose dehydrogen ase-Methode mit der Hexokinase-Methode

Die Proben stammten aus der täglichen Routine unserer Kliniken. Die Gefäße für die Blutentnahme enthielten Ammoniumheparinat, wurden nach der Blutentnahme sofort zentrifugiert und das Plasma abgehebert. Das Verhältnis Männer zu Frauen beträgt etwa $1: 1$, und das Alter der Patienten liegt zwischen 18 und 80 Jahren. Abbildung 5 zeigt das Korrelationsdiagramm.

Die Hämodialysepatienten wurden im Methodenvergleich gesondert behandelt (Korrelationskoeffizient $r=0,994, N=41$ ). Frühere Untersuchungen an anderen Parametern zeigten, daß Hämodialysepatienten of tmals besondere Veränderungen ihrer Blutzusammensetzung aufwiesen.

\section{Diskussion}

Gemäß den vorliegenden Resultaten kann diese Methode als geeignet gelten, um auf dem GSA II routinemäßig gefahren zu werden. Die Spezifität läßt nach unserer Auffassung kaum zu wünschen übrig. Die mit der Glucosedehydrogenase-Methode gemachten Erfahrungen gelten nicht für die Hexokinase-Methode, die auch hier absolut spezifisch ist. Nach eigenen Beobachtungen liefert die Glucoseoxidase-Methode, wie sie im Beckman-

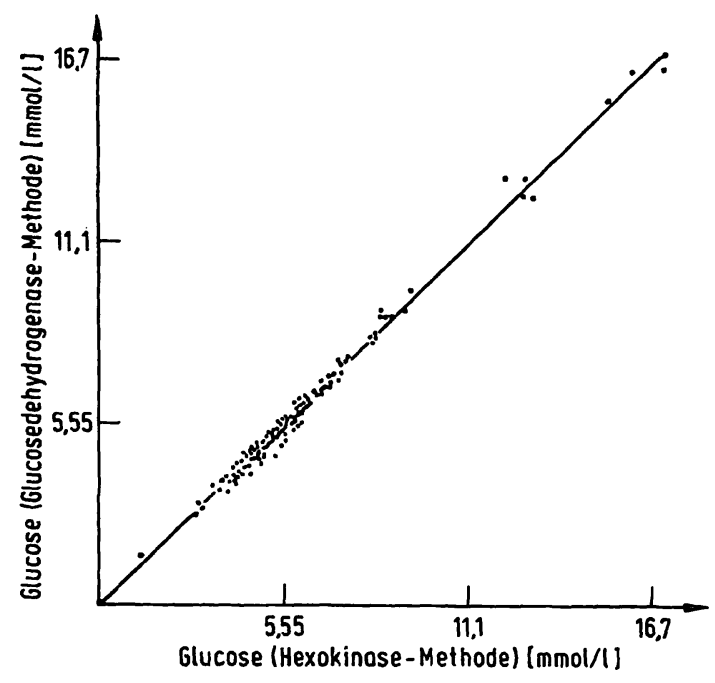

Abb. 5. Vergleich zwischen der Hexokinase-Methode und Glucosedehydrogenase-Methode (Patienten der medizinischen und chirurgischen Klinik sowie Hämodialysepatienten).

$$
\begin{aligned}
y & =-0,13+1,009 x, \quad x=0,997 \\
\bar{x}=6,23, \bar{y}=6,18, & s_{y x}= \pm 0,019 \quad N=159
\end{aligned}
$$

Glucoseanalyzer angewendet wird, auch falsche Werte, wenn 2-Desoxyglucose vorhanden ist. Wir konnten mit dieser Glucosedehydrogenase-Methode ca. 80\% Reagenzienkosten einsparen. Dies gilt nicht nur für den Kauf des Materials, sondern auch für die Herstellung der Lösung: es wird nur ein einziges, einfach herzustellendes Reagenz benötigt.

Als neue Referenzmethode eignet sich das Glucosedehydrogenase-System nicht; hierzu reicht die Spezifität nicht aus.

\section{Danksagung}

Herrn Prof. Dr. M. Schmid danke ich für die kritische Durchsicht des Manuskriptes und Frl. G. Bächtold für das Schreiben der endgültigen Fassung.

Der Firma Merck, Darmstadt, danken wir für die kostenlose Überlassung von Reagenzien für die GlucosedehydrogenaseMethode.

\section{Literatur}

1. Metzger, R. P., Wilcox, S. S. \& Wịck, A. N. (1964), J. Biol. Chem. 239, 1769-1772.

2. Greiner, R. (1973), diese Z. 11, 76-86.

3. Bergmeyer, H. U. (1970), Methoden der enzymatischen Analyse, S. 273-279 (Bergmeyẹ, H. U., ed.), Verlag Chemie, Weinheim/Bergstrạße.

4. Bestimmung der Glucose in Plasma, Serum und Urin mit einer Hexokinase/Glucose-6-PhosphatdehydrogenaseMethode. Handbook Greiner Electrọnic Selective Analyzer Iİ, 1972.

5. Richterich, R. (1968), Klinische Chemie in Theorie und Praxis, 2. Auflage, S. 68-71, Karger, Basel.
6. Marbach, E. P., McLean, M., Scharn, Marylin \& Tom Jones (1975), Clin. Chem. 21, 1810-1812.

7. Kohler, K. (1963), Intern. Technicon Symposium, Automation in der Analytischen Chemie, S. 123-127.

8. Bạnauch, D., Brümmer, W., Ebeling, H., Metz, H., Rindfrey, H. \& Lang, H. (1975), diese Z. 13, 101-107.

9. Duke, W. W., Hirschowitz, B. I. \& Sachs, G. (1965), Lancet, II, 871-875.

10. Gogh, A. L. \& Keddie, N. C. (1975), Gut, 16, 171-175.

11. Walter, A. M. \& Heilmeyer, L. (1975), Antibiotika Fibel, G. Thieme Verlag, Stuttgart, S. 272-292. 
